INVESTIGACIÓN

Recibido: 23/05/2020 --- Aceptado: 06/07/2020 --- Publicado: 12/03/2021

\title{
ANÁLISIS SOBRE EL IMPACTO DEL AULA INVERTIDA Y LA TECNOLOGÍA EN EL PROCESO EDUCATIVO SOBRE EL DISEÑO DE LA COMUNICACIÓN GRÁFICA
}

\section{Analysis about the impact of flipped classroom and technology in the educational process on the Design of Graphic Communication}

8in Ricardo-Adán Salas-Rueda1. Universidad Nacional Autónoma de México. México. ricardo.salas@icat.unam.mx

Ana-Libia Eslava-Cervantes. Universidad Nacional Autónoma de México. México. libia.eslava@icat.unam.mx

Estefanía Prieto-Larios. Universidad Nacional Autónoma de México. México. estefaníaprietolarios@gmail.com

Agradecimientos. Este producto de investigación recibió el apoyo del proyecto "El Aula del Futuro". Se agradece la participación de los académicos: Dra. Clara Alvarado Zamorano, Dr. Gustavo De la Cruz Martínez, M. en P. Jesús Ramírez Ortega, M. A. Ricardo Castañeda Martínez y M. en I. Antonio M. Garcés Madrigal.

\section{RESUMEN}

El objetivo de esta investigación mixta es analizar el impacto del aula invertida en el proceso educativo sobre el Diseño de la Comunicación Gráfica. La muestra está compuesta por 20 alumnos que cursaron la asignatura Expresión Formal I en una universidad pública de la Ciudad de México durante el ciclo escolar 2020. Esta investigación propone la consulta de videos antes de la clase y el uso de la aplicación Sketchbook durante la sesión presencial por medio de los dispositivos móviles. La aplicación Sketchbook permite analizar las imágenes del rostro humano, dibujar y comprobar las dimensiones de los objetos por medio de los dispositivos móviles. En la casa, los estudiantes consultaron los videos sobre la aplicación Sketchbook con la finalidad de aprender el uso de esta herramienta tecnológica. Los resultados del aprendizaje automático (regresión lineal) indican que el uso de la aplicación Sketchbook y los dispositivos móviles en el proceso de aprendizaje influyen

\footnotetext{
1 Ricardo-Adán Salas-Rueda: Doctor en Diseño de Nuevas Tecnologías. Investigador de tiempo completo en el Instituto de Ciencias Aplicadas y Tecnología, Universidad Nacional Autónoma de México. Investigador Nacional SNI nivel 1 (2019-2021). http:/ / orcid.org/0000-0002-4188-4610
} 
Salas-Rueda, R. A., Eslava-Cervantes, A. L. y Prieto-Larios, E.

Análisis sobre el impacto del aula invertida y la tecnología en el proceso educativo sobre el diseño de la comunicación gráfica

positivamente el rol activo de los estudiantes. El aula invertida permite la construcción de nuevos espacios educativos donde los alumnos participan activamente durante el proceso educativo. Por último, el aula invertida es un modelo pedagógico que está transformando la organización y realización de las actividades en el Siglo XXI con el apoyo de la tecnología.

PALABRAS CLAVE: Aula invertida - Tecnología - Educación - Enseñanza - TIC Tecnología educativa - Aprendizaje.

\section{ABSTRACT}

The objective of this mixed research is to analyze the impact of flipped classroom in the educational process on the Design of Graphic Communication. The sample is made up of 20 students who took the Formal Expression I course at a public university in Mexico City during the 2020 school year. This research proposes the consultation of the videos before the class and use of the Sketchbook application during the face-to-face session through the mobile devices. The Sketchbook application allows analyzing the images of the human face, draw and check the dimensions of the objects through the mobile devices. At home, the students consulted the videos about the Sketchbook application in order to learn the use of this technological tool. The results of machine learning (linear regression) indicate that the use of the Sketchbook application and mobile devices in the learning process positively influence the active role of the students. Flipped classroom allows the construction of new educational spaces where students actively participate during the educational process. Finally, flipped classroom is a pedagogical model that is transforming the organization and implementation of the activities in the 21st century with the support of technology.

KEY WORDS: Flipped classroom - Technology - Education - Teaching - ICT Educational technology - Learning.

\section{ANÁLISE SOBRE O IMPACTO DA SALA DE AULA INVERTIDA E DA TECNOLOGIA NO PROCESSO EDUCACIONAL NO DESIGN DE COMUNICAÇÃO GRÁFICA}

\section{RESUMO}

O objetivo desta pesquisa mista é analisar o impacto da sala de aula invertida no processo educativo sobre o design de comunicação Gráfica. A amostra é composta por 20 estudantes que cursaram a matéria Expresión Formal I em uma universidade pública da Cidade do México durante o ciclo letivo de 2020. Esta pesquisa propõe a consulta de vídeos antes da aula e o uso do aplicativo Sketchbook durante a sessão presencial por meio de dispositivos móveis. O aplicativo Sketchbook permite analisar as imagens do rosto humano, desenhar e verificar as dimensões dos objetos através de dispositivos móveis. Em casa, os alunos consultaram os vídeos sobre o aplicativo 
Salas-Rueda, R. A., Eslava-Cervantes, A. L. y Prieto-Larios, E.

Análisis sobre el impacto del aula invertida y la tecnología en el proceso educativo sobre el diseño de la comunicación gráfica

Sketchbook com a finalidade de aprender a usar esta ferramenta tecnológica. Os resultados do aprendizado automático (regressão linear) demonstram que o uso do aplicativo Sketchbook e os dispositivos móveis no processo de aprendizado influenciam positivamente no papel ativo dos alunos. A sala de aula invertida permite a construção de novos espaços educativos onde os estudantes participam ativamente durante o processo educativo. Por último, a sala de aula invertida é um modelo pedagógico que está transformando a organização e realização das atividades no século XXI com o apoio da tecnologia.

PALAVRAS CHAVE: Sala de aula Invertida - Tecnologia - Educação - Ensino - TIC - Tecnologia educativa - Aprendizado.

\section{Cómo citar el artículo:}

Salas-Rueda, R. A., Eslava-Cervantes, A. L. y Prieto-Larios, E. (2021). Análisis sobre el impacto del aula invertida y la tecnología en el proceso educativo sobre el diseño de la comunicación gráfica. Vivat Academia. Revista de Comunicación, 154, 25-39. http:/ / doi.org/10.15178/va.2021.154.e1238

http://www.vivatacademia.net/index.php/vivat/article/view/1238

\section{INTRODUCCIÓN}

El aula invertida es una modalidad blended learning que facilita la participación de los estudiantes dentro y fuera del salón de clases (Hew \& Lo, 2018). El uso de este modelo pedagógico se está incrementado en las universidades debido a que los docentes buscan innovar el proceso educativo con el apoyo de las herramientas tecnológicas y plataformas web educativas (Busebaia \& John, 2020; Hew \& Lo, 2018; Rui et al., 2017). De hecho, el aula invertida reemplaza las actividades realizadas en el salón de clases con la elaboración de tareas individuales y colaborativas en la casa (Bakla, 2018; Uskoković, 2018). De hecho, este modelo pedagógico permite la creación de espacios educativos que incrementan la satisfacción y motivación de los estudiantes durante el proceso de enseñanza-aprendizaje (Busebaia \& John, 2020; Tague \& Czocher, 2016).

Antes de la sesión presencial, los alumnos consultan los recursos multimedia desde cualquier lugar (Rui et al., 2017; Tague \& Czocher, 2016). En el salón de clases, los docentes organizan creativas actividades individuales y colaborativas que facilitan el rol activo de los estudiantes (Barbour \& Schuessler, 2019; Tague \& Czocher, 2016). Los docentes tienen la posibilidad de actualizar los cursos, innovar las actividades escolares dentro y fuera del salón y organizar nuevos espacios virtuales educativos por medio del aula invertida (He, Holton, \& Farkas, 2018; SalasRueda, 2020; Zainuddin, 2018). De hecho, este modelo pedagógico favorece la participación activa antes, durante y después de la clase (Barbour \& Schuessler, 2019; Hermsen, Mokadam, \& Verrier, 2019). 
Salas-Rueda, R. A., Eslava-Cervantes, A. L. y Prieto-Larios, E.

Análisis sobre el impacto del aula invertida y la tecnología en el proceso educativo sobre el diseño de la comunicación gráfica

El objetivo de esta investigación mixta es analizar el impacto del aula invertida en el proceso educativo sobre el Diseño de la Comunicación Gráfica. Por consiguiente, las preguntas de investigación son:

- ¿Cuál es el impacto sobre el uso de la aplicación Sketchbook bajo la modalidad del aula invertida?

- ¿Cuál es el impacto sobre el uso de los dispositivos móviles bajo la modalidad del aula invertida?

- ¿Cuáles son las percepciones de los estudiantes sobre el uso de la tecnología bajo la modalidad del aula invertida?

\subsection{Aula invertida}

En el Siglo XXI, el aula invertida está cambiando radicalmente las funciones de los participantes del proceso educativo durante la organización y realización de las actividades escolares (Bakla, 2018; Busebaia \& John, 2020). Por ejemplo, los docentes buscan nuevos modelos pedagógicos que faciliten la asimilación de conocimiento y el desarrollo de las competencias en los estudiantes (Rotellar \& Cain, 2016). El aula invertida con el apoyo de las Tecnologías de la Información y Comunicación (TICs) mejoró las condiciones de enseñanza-aprendizaje en los cursos de Medicina (Hew \& Lo, 2018; Rui et al., 2017), Informática (Salas-Rueda, 2020), Pediatría (Busebaia \& John, 2020) y Matemáticas (Salas-Rueda, 2018; Tague \& Czocher, 2016).

Los docentes utilizan los modelos pedagógicos y las nuevas tecnologías en el campo educativo con la finalidad de mejorar el proceso de aprendizaje (Hew \& Lo, 2018; Salas-Rueda, 2015). En el curso de Medicina, el aula invertida facilita la participación activa de los estudiantes antes de la sesión presencial por medio de la consulta de videos y lecturas digitales (Hew \& Lo, 2018). Al inicio de la sesión presencial, la resolución de exámenes en línea mejoró el rendimiento académico (Hew \& Lo, 2018). Incluso, los alumnos de medicina tienen el papel principal en el proceso educativo debido a la realización de actividades colaborativas en el salón de clases (Hew \& Lo, 2018).

El aula invertida promueve el uso de las herramientas tecnológicas, el software educativo y las aplicaciones web con la finalidad de facilitar el proceso de aprendizaje y desarrollar las competencias de los estudiantes (Salas-Rueda, 2018). Fuera del salón de clases, los alumnos del curso Matemáticas Computacionales consultaron los videos para adquirir los conocimientos sobre el uso de la aplicación FreeDFD (Salas-Rueda, 2018). En el salón de clases, los alumnos resolvieron los ejercicios de forma colaborativa y comprobaron los resultados por medio de la aplicación FreeDFD (Salas-Rueda, 2018). Por último, los alumnos del curso Matemáticas Computacionales resolvieron las prácticas de laboratorio después de la sesión presencial por medio de la aplicación FreeDFD (Salas-Rueda, 2018).

El aula invertida ha mejorando las condiciones de enseñanza-aprendizaje en todos los niveles educativos (Bakla, 2018; Rui et al., 2017; Tague \& Czocher, 2016). En el

Vivat Academia. Revista de Comunicación. 2021, nº 154, 25-39 
Salas-Rueda, R. A., Eslava-Cervantes, A. L. y Prieto-Larios, E.

Análisis sobre el impacto del aula invertida y la tecnología en el proceso educativo sobre el diseño de la comunicación gráfica

curso de Ecuaciones Diferenciales, los alumnos consultaron las animaciones web en la casa para facilitar la asimilación del conocimiento (Tague \& Czocher, 2016). Las animaciones web sobre las ecuaciones web contienen diversos cuestionarios que permiten evaluar el rendimiento académico de los estudiantes (Tague \& Czocher, 2016). La resolución de los ejercicios y la realización de los foros de discusión en el salón de clases facilitaron la participación activa de los estudiantes y mejoraron el rendimiento académico (Tague \& Czocher, 2016).

En el campo de la Salud, los docentes consideran que la interpretación del electrocardiograma es uno de los temas más difíciles de aprender durante la realización de los diagnósticos clínicos (Rui et al., 2017). Por consiguiente, el aula invertida representa una excelente alternativa para mejorar el proceso de enseñanzaaprendizaje en el área de la Medicina (Rui et al., 2017). Los alumnos de Medicina consultaron los recursos multimedia, los capítulos de libros electrónicos y las lecturas digitales sobre el funcionamiento del corazón en la casa (Rui et al., 2017). En el salón de clases, los foros de discusión y el trabajo colaborativo permitió el rol activo de los estudiantes (Rui et al., 2017). Los resultados del aula invertida son el mejoramiento del rendimiento académico, incremento de la satisfacción y desarrollo de las habilidades en los estudiantes (Rui et al., 2017).

Hoy en día, los docentes pueden rediseñar los cursos por medio del aula invertida (Busebaia \& John, 2020). En el curso de Pediatría, los alumnos revisaron las presentaciones digitales y consultaron los videos en la casa (Busebaia \& John, 2020). Durante la sesión presencial, la resolución de casos de estudio, realización de foros de discusión y presentación de los temas facilitaron el rol activo de los estudiantes (Busebaia \& John, 2020). Después la sesión presencial, los alumnos de Pediatría resolvieron los exámenes en línea (Busebaia \& John, 2020).

El aula invertida permite organizar y realizar nuevas actividades antes, durante y después de la clase por medio del uso de las aplicaciones web y el trabajo colaborativo (Salas-Rueda, 2020). Antes de la sesión presencial, los alumnos de Informática consultaron los videos sobre el uso de la base de datos para insertar, actualizar, eliminar y consultar la información (Salas-Rueda, 2020). Durante la sesión presencial, el trabajo colaborativo y el uso de la aplicación MySQL facilitó la asimilación del conocimiento sobre el uso de las base de datos y desarrolló las habilidades tecnológicas de los estudiantes (Salas-Rueda, 2020). Después de la sesión presencial, los alumnos de Informática tuvieron una participación activa por medio de la realización de las prácticas de laboratorio (Salas-Rueda, 2020). El uso del aula invertida en el campo de la Informática incrementó la motivación y el rendimiento de los estudiantes por medio de la realización de las actividades antes, durante y después de la clase (Salas-Rueda, 2020).

Uskoković (2018) propone que las actividades del aula invertida incluyan la realización de foros de discusión y exámenes en línea antes de la sesión presencial, la realización de foros de discusión durante la sesión presencial y la elaboración de tareas en forma colaborativa después de la sesión presencial con la finalidad de

Vivat Academia. Revista de Comunicación. 2021, n 154, 25-39 
Salas-Rueda, R. A., Eslava-Cervantes, A. L. y Prieto-Larios, E.

Análisis sobre el impacto del aula invertida y la tecnología en el proceso educativo sobre el diseño de la comunicación gráfica

facilitar la asimilación del conocimiento y desarrollar las competencias en los estudiantes.

El aula invertida mejora las condiciones de enseñanza-aprendizaje con el apoyo de las herramientas tecnológicas (Busebaia \& John, 2020; He, Holton, \& Farkas, 2018; Tague \& Czocher, 2016). De hecho, las instituciones educativas utilizan este modelo pedagógico para organizar creativas actividades antes, durante y después de la sesión presencial (Barbour \& Schuessler, 2019; Busebaia \& John, 2020; Uskoković, 2018).

\section{OBJETIVOS}

El objetivo general de esta investigación mixta es analizar el impacto del aula invertida en el proceso educativo sobre el Diseño de la Comunicación Gráfica. La muestra está compuesta por 20 alumnos que cursaron la asignatura Expresión Formal I en una universidad pública de la Ciudad de México durante el ciclo escolar 2020.

Los objetivos particulares de esta investigación son (1) analizar el impacto sobre el uso de la aplicación Sketchbook bajo la modalidad del aula invertida (2) analizar el impacto sobre el uso de los dispositivos móviles bajo la modalidad del aula invertida y (3) analizar las percepciones de los estudiantes sobre el uso de la tecnología bajo la modalidad del aula invertida.

\section{METODOLOGÍA}

A continuación, se presenta la metodología utilizada en esta investigación mixta por medio de las secciones de Procedimiento, Recolección de datos y Análisis de datos.

\subsection{Procedimiento}

El profesor de la asignatura Expresión Formal I cursó el Diplomado Aula del Futuro 2020 impartido por la Universidad Nacional Autónoma de México (UNAM) con el propósito de mejorar las condiciones de enseñanza-aprendizaje. Este Diplomado utiliza el modelo tecno-pedagógico propuesto por Gamboa-Rodríguez (2015) para transformar el proceso educativo por medio de la pedagogía y la tecnología.

El Diplomado Aula del Futuro 2020 permitió transformar las actividades de la asignatura Expresión Formal I antes y durante la sesión presencial bajo la modalidad del aula invertida.

Esta investigación propone la consulta de videos antes de la clase y el uso de la aplicación Sketchbook durante la sesión presencial por medio de los dispositivos móviles. En la casa, los estudiantes de la Licenciatura en Diseño de la Comunicación

Vivat Academia. Revista de Comunicación. 2021, nº 154, 25-39 
Salas-Rueda, R. A., Eslava-Cervantes, A. L. y Prieto-Larios, E.

Análisis sobre el impacto del aula invertida y la tecnología en el proceso educativo sobre el diseño de la comunicación gráfica

Gráfica consultaron los videos sobre la aplicación Sketchbook con la finalidad de aprender el uso de esta herramienta tecnológica.

Durante la sesión presencial, los alumnos de la asignatura Expresión Formal I utilizaron la aplicación Sketchbook para facilitar la asimilación del conocimiento sobre el trazo del rostro humano. En el salón de clases, los alumnos analizaron las imágenes del rostro humano, dibujaron y comprobaron sus dimensiones en los dispositivos móviles por medio de la aplicación Sketchbook. La Figura 1 muestra el modelo de aceptación tecnológica utilizado en esta investigación.

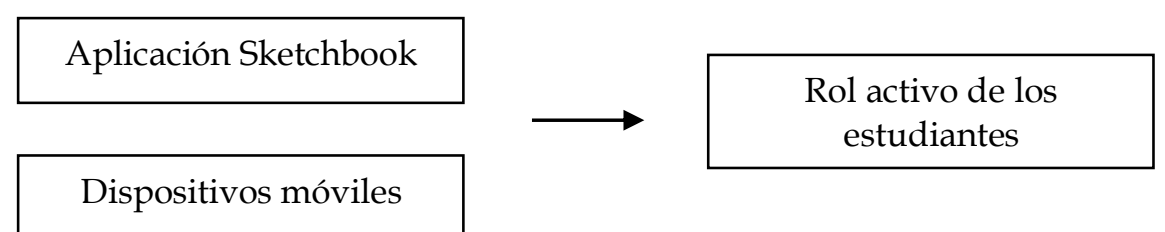

Figura 1: Modelo de aceptación tecnológica propuesto.

Fuente: Elaboración propia.

Las hipótesis de investigación relacionados con el uso del aula invertida en el proceso de enseñanza-aprendizaje son:

- Hipótesis 1 (H1): El uso de la aplicación Sketchbook en el proceso de aprendizaje influye positivamente el rol activo de los estudiantes bajo la modalidad del aula invertida

- Hipótesis 2 (H2): El uso de los dispositivos móviles en el proceso de aprendizaje influye positivamente el rol activo de los estudiantes bajo la modalidad del aula invertida

\subsection{Recolección de datos}

La recolección de datos se realizó en una universidad pública de la Ciudad de México durante el mes de Febrero del 2020. El instrumento de medición es un cuestionario que consta de 5 preguntas cerradas y 1 pregunta abierta (Ver Tabla 1).

Tabla 1. Cuestionario sobre el uso del aula invertida.

\begin{tabular}{|c|c|c|c|c|c|c|}
\hline No. & Variable & Dimensión & Pregunta & Respuesta & $\mathrm{n}$ & $\%$ \\
\hline \multirow{6}{*}{1} & \multirow{6}{*}{$\begin{array}{c}\text { Características } \\
\text { de los } \\
\text { estudiantes }\end{array}$} & \multirow[t]{2}{*}{ Sexo } & \multirow[t]{2}{*}{ 1. ¿Cuál es tu sexo? } & Hombre & 8 & $40.00 \%$ \\
\hline & & & & Mujer & 12 & $60.00 \%$ \\
\hline & & \multirow{4}{*}{ Edad } & \multirow{4}{*}{ 2. ¿Cuál es tu edad? } & 18 años & 8 & $40.00 \%$ \\
\hline & & & & 19 años & 6 & $30.00 \%$ \\
\hline & & & & 20 años & 1 & $5.00 \%$ \\
\hline & & & & $>20$ años & 5 & $25.00 \%$ \\
\hline \multirow{2}{*}{2} & \multirow{2}{*}{$\begin{array}{c}\text { Aula } \\
\text { invertida }\end{array}$} & \multirow{2}{*}{$\begin{array}{l}\text { Aplicación } \\
\text { Sketchbook }\end{array}$} & \multirow{2}{*}{$\begin{array}{lcc}3 . \quad \text { La } & \text { aplicación } \\
\text { Sketchbook } & \text { mejoró mi }\end{array}$} & & & \\
\hline & & & & Mucho (1) & 13 & $65.00 \%$ \\
\hline
\end{tabular}

Vivat Academia. Revista de Comunicación. 2021, nº 154, 25-39 
Salas-Rueda, R. A., Eslava-Cervantes, A. L. y Prieto-Larios, E.

Análisis sobre el impacto del aula invertida y la tecnología en el proceso educativo sobre el diseño de la comunicación gráfica

\begin{tabular}{|c|c|c|c|c|c|c|}
\hline & & & proceso de aprendizaje & Bastante (2) & 7 & $35.00 \%$ \\
\hline & & & & Poco (3) & 0 & $0.00 \%$ \\
\hline & & & & Muy poco (4) & 0 & $0.00 \%$ \\
\hline & & & 4. $\quad \mathrm{El}$ & Murb (1) & 11 & $5500 \%$ \\
\hline & & Dispositivos & dispositivos móviles & Bastante (2) & 9 & $4500 \%$ \\
\hline & & móviles & mejoró mi proceso de & Poco (3) & 0 & $0.00 \%$ \\
\hline & & & & Muy poco (4) & 0 & $0.00 \%$ \\
\hline & & & 5 Las & Mucho (1) & 13 & $65.00 \%$ \\
\hline & & Rol activo & durante la clase & Bastante (2) & 7 & $35.00 \%$ \\
\hline & & & facilitaron mi rol activo & Poco (3) & 0 & $0.00 \%$ \\
\hline & & & & Muy poco (4) & 0 & $0.00 \%$ \\
\hline 3 & $\begin{array}{c}\text { Percepción de } \\
\text { los } \\
\text { estudiantes }\end{array}$ & Tecnología & $\begin{array}{l}\text { 6. ¿Cuál es tu opinión } \\
\text { sobre la incorporación } \\
\text { de la tecnología en las } \\
\text { actividades escolares? }\end{array}$ & Pregunta abierta & - & - \\
\hline
\end{tabular}

Fuente: Elaboración propia.

\subsection{Análisis de datos}

Esta investigación utiliza la aplicación Rapidminer para evaluar las hipótesis de investigación sobre el uso del aula invertida en el proceso educativo sobre el Diseño de la Comunicación Gráfica. La sección de entrenamiento $(60 \%, 70 \%$ y $80 \%$ de la muestra) permite calcular las regresiones lineales sobre la aplicación Sketchbook y el uso de los dispositivos móviles. La sección de evaluación $(40 \%, 30 \%$ y 20\% de la muestra) permite identificar la exactitud de estas regresiones lineales por medio del error cuadrado.

Por otro lado, la aplicación Wordclouds permite analizar las percepciones de los estudiantes sobre el uso de la tecnología en el aula invertida por medio de la nube de palabras.

\section{RESULTADOS Y DISCUSIÓN}

A continuación se presentan los resultados del aula invertida en el proceso educativo sobre el Diseño de la Comunicación Gráfica. Las actividades durante la clase facilitaron mucho $(n=13,65.00 \%)$ y bastante $(n=7,35.00 \%)$ el rol activo de los estudiantes (Ver Tabla 1). Asimismo, los resultados del aprendizaje automático (regresión lineal) indican que el uso de la aplicación Sketchbook y los dispositivos móviles en el proceso de aprendizaje influyen positivamente el rol activo de los estudiantes bajo la modalidad del aula invertida (Ver Tabla 2).

Tabla 2. Resultados del aprendizaje automático.

\begin{tabular}{|l|l|l|l|c|}
\hline Hipótesis & Entrenamiento & Regresión lineal & Conclusión & $\begin{array}{c}\text { Error } \\
\text { cuadrado }\end{array}$ \\
\hline
\end{tabular}


Salas-Rueda, R. A., Eslava-Cervantes, A. L. y Prieto-Larios, E.

Análisis sobre el impacto del aula invertida y la tecnología en el proceso educativo sobre el diseño de la comunicación gráfica

\begin{tabular}{|l|c|c|c|c|}
\hline \multirow{2}{*}{$\begin{array}{l}\text { H1: Uso de la aplicación } \\
\text { Sketchbook } \rightarrow \text { Rol activo de }\end{array}$} & $60 \%$ & $\mathrm{y}=0.111 \mathrm{x}+1.114$ & Acepta: 0.111 & 0.275 \\
\cline { 2 - 5 } los estudiantes & $70 \%$ & $\mathrm{y}=0.151 \mathrm{x}+1.030$ & Acepta: 0.151 & 0.358 \\
\cline { 2 - 5 } & $80 \%$ & $\mathrm{y}=0.218 \mathrm{x}+0.963$ & Acepta: 0.218 & 0.356 \\
\hline \multirow{2}{*}{$\begin{array}{l}\text { H2: Uso de los dispositivos } \\
\text { móviles } \rightarrow \text { Rol activo de los } \\
\text { estudiantes }\end{array}$} & $60 \%$ & $\mathrm{y}=0.166 \mathrm{x}+1.000$ & Acepta: 0.166 & 0.309 \\
\cline { 2 - 5 } & $70 \%$ & $\mathrm{y}=0.208 \mathrm{x}+0.916$ & Acepta: 0.208 & 0.424 \\
\cline { 2 - 6 } & $80 \%$ & $\mathrm{y}=0.063 \mathrm{x}+1.158$ & Acepta: 0.063 & 0.419 \\
\hline
\end{tabular}

Fuente: Elaboración propia.

\subsection{Uso de la aplicación Sketchbook}

La aplicación Sketchbook mejoró mucho $(\mathrm{n}=13,65.00 \%)$ y bastante $(\mathrm{n}=7,35.00 \%)$ el proceso de aprendizaje (Ver Tabla 1). Asimismo, los resultados del aprendizaje automático con $60 \%$ (0.111), 70\% (0.151) y 80\% (0.218) de entrenamiento indican que la Hipótesis 1 es aceptada (Ver Tabla 2). Por lo tanto, el uso de la aplicación Sketchbook en el proceso de aprendizaje influye positivamente el rol activo de los estudiantes bajo la modalidad del aula invertida.

\subsection{Uso de dispositivos móviles}

El uso de dispositivos móviles mejoró mucho $(\mathrm{n}=11,55.00 \%)$ y bastante $(\mathrm{n}=9$, $45.00 \%$ ) el proceso de aprendizaje (Ver Tabla 1). Asimismo, los resultados del aprendizaje automático con $60 \%(0.166), 70 \%(0.208)$ y $80 \%(0.063)$ de entrenamiento indican que la Hipótesis 2 es aceptada (Ver Tabla 2). Por lo tanto, el uso de los dispositivos móviles en el proceso de aprendizaje influye positivamente el rol activo de los estudiantes bajo la modalidad del aula invertida.

La Gráfica 1 muestra los resultados sobre el uso de la aplicación Sketchbook y los dispositivos móviles en el aula invertida.

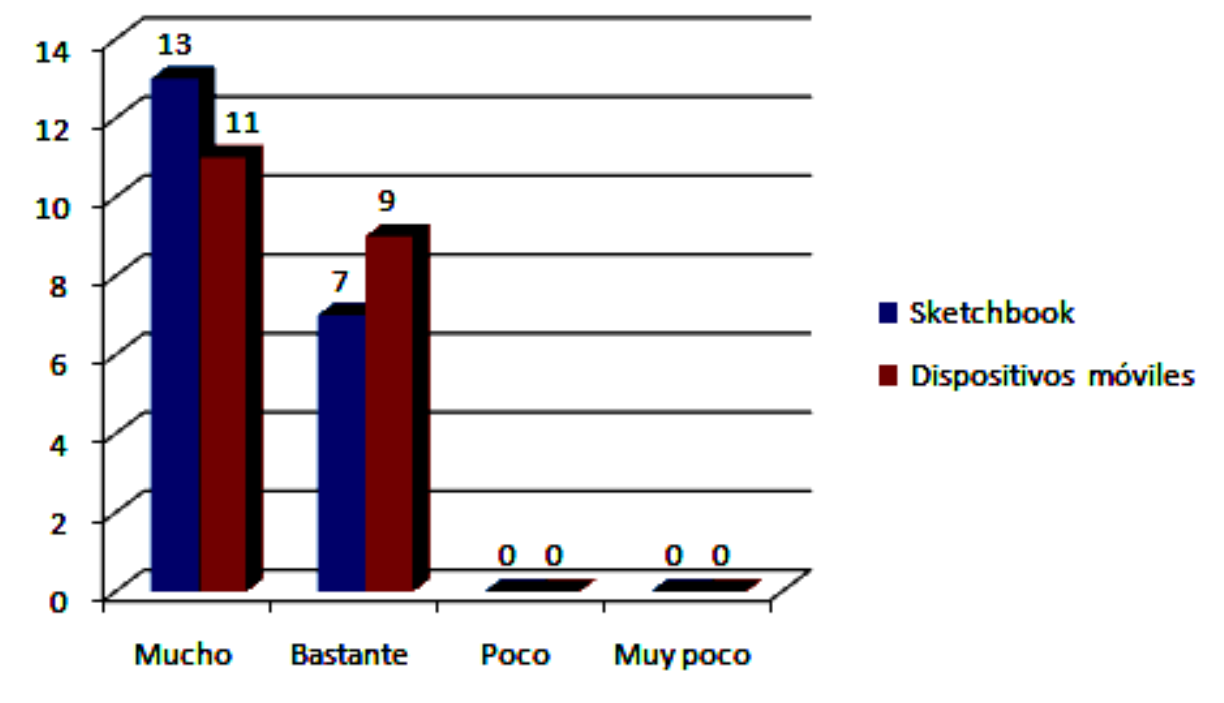

Gráfica 1. Uso de la aplicación Sketchbook y los dispositivos móviles en el aula invertida. 
Salas-Rueda, R. A., Eslava-Cervantes, A. L. y Prieto-Larios, E.

Análisis sobre el impacto del aula invertida y la tecnología en el proceso educativo sobre el diseño de la comunicación gráfica

Fuente: Elaboración propia.

\subsection{Percepciones de los estudiantes}

Los avances tecnológicos están cambiando la forma de interactuar entre los usuarios y los contenidos. Los alumnos de la Licenciatura en Diseño de la Comunicación Gráfica piensan que el uso de la aplicación Sketchbook en el aula invertida es muy útil durante el proceso de enseñanza-aprendizaje.

"Es algo muy útil porque cada vez se usa más la tecnología en la vida e incorporarla en el aprendizaje facilita más las cosas y ayuda a estar más actualizados" (Estudiante 18, mujer, > 20 años).

"Se me hizo algo muy útil, ya que puedes ver con más certeza detalles que normalmente no alcanzamos a percibir" (Estudiante 19, mujer, 18 años).

El aula invertida permite la organización de creativas actividades durante el proceso de enseñanza-aprendizaje. En particular, la aplicación Sketchbook facilita la asimilación del conocimiento por medio del dibujo y uso de los objetos.

"Que con su uso nos ayuda a tener más facilidad y precisión a la hora del dibujo, así que es una muy buena opción para el diseño" (Estudiante 4, hombre, 18 años).

"Facilita por mucho varios aspectos como la precisión, la limpieza, la calidad de línea y el trabajo en si" (Estudiante 10, hombre 19 años).

Los docentes utilizan el aula invertida para construir nuevos espacios educativos donde los alumnos participan activamente dentro y fuera del salón. Por ejemplo, los alumnos mencionan que la aplicación Sketchbook permite la realización de actividades innovadoras y creativas.

"Se me hace algo muy acertado, hace que sea más fácil entender y le da un cambio a lo típico, lo que hace que sea más atractivo" (Estudiante 13, hombre, $>20$ años).

“La utilización de nuevas herramientas tecnológicas nos permite involucrarnos más en cualquier ámbito del dibujo y conocer cosas y técnicas de una manera más práctica sin tener que usar materiales físicos" (Estudiante 15, hombre, 19 años).

La incorporación de la tecnología en las actividades escolares facilita el aprendizaje y el desarrollo de las habilidades. La aplicación Sketchbook facilita la realización de los trazos y el manejo de imágenes.

“Me parece más fácil ya que facilitan varios trazos que a mano son más difíciles de hacer y que puedes utilizar cualquier imagen sin necesidad de imprimir entre otras herramientas" (Estudiante 9, hombre, 20 años).

"Es necesario y considero que no todos tienen las habilidades motrices para hacer algo en una hoja, pero si en un dispositivo" (Estudiante 20, mujer, 19 años).

Vivat Academia. Revista de Comunicación. 2021, nº 154, 25-39 

diseño de la comunicación gráfica

Los modelos pedagógicos como el aula invertida y el uso de las herramientas tecnológicas mejoran las condiciones de enseñanza-aprendizaje. En la asignatura de Expresión Formal I, los alumnos consideran que el uso de la aplicación Sketchbook incrementa la motivación de los estudiantes.

"Son un gran apoyo para realizar más ejercicios y de manera más practica sobre temas que veamos. Hace una clase más dinámica" (Estudiante 16, mujer, 18 años).

"Que afecta de manera positiva en nuestro aprendizaje, ya que hace que nos llame más la atención y nos hace tener, o mejor dicho, nos motiva a aprender y a usar la tecnología en nuestro favor" (Estudiante 17, mujer, 19 años).

La nube de palabras muestra que tecnología, aprendizaje, útil, facilita, importante, actividades y ayuda son las palabras que más se repiten (Ver Figura 2).

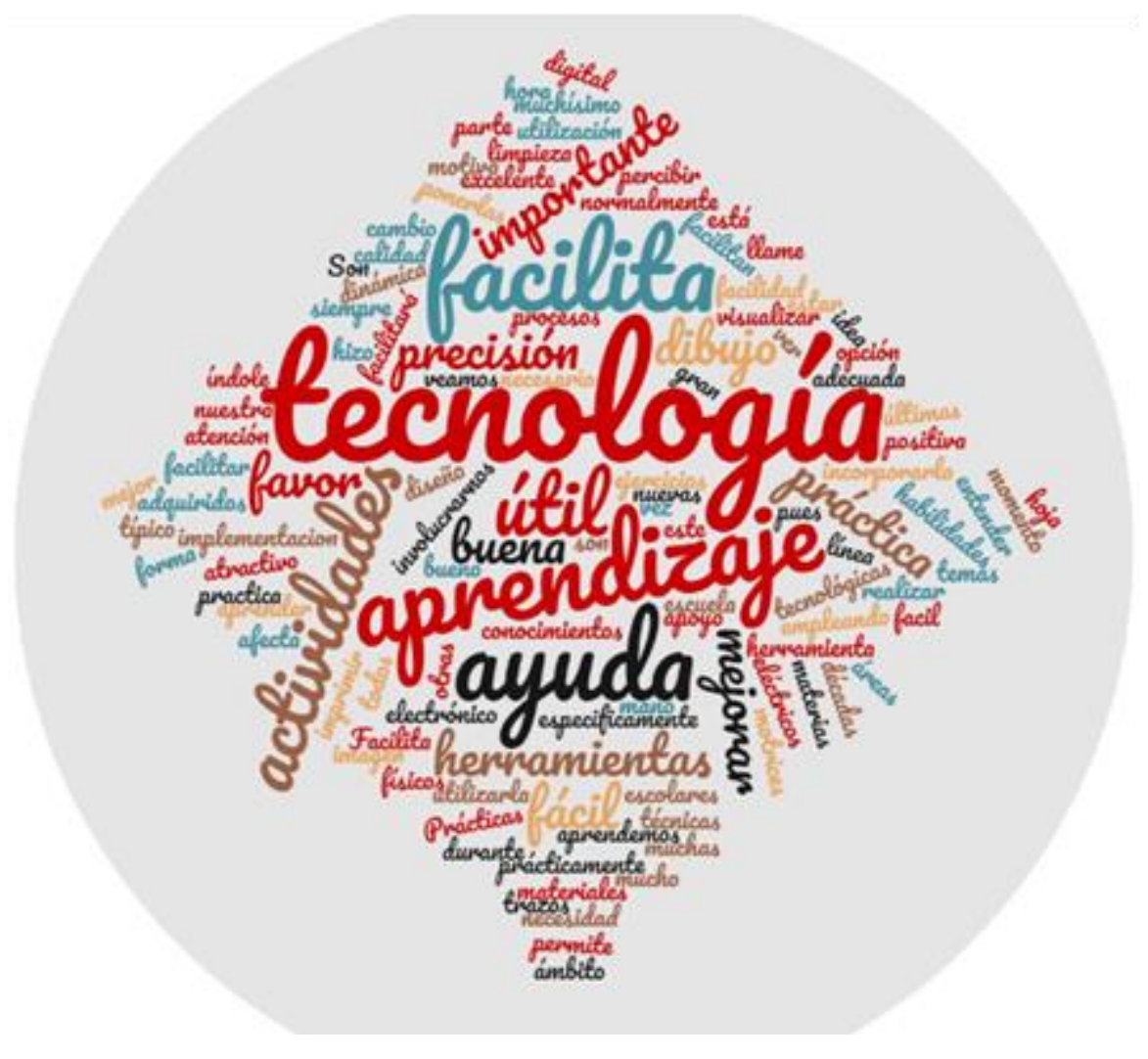

Figura 2. Nube de palabras sobre el uso de la tecnología en el aula invertida.

Fuente: elaboración propia.

\subsection{Discusión}

Los avances tecnológicos junto con los modelos pedagógicos están cambiando la forma de interactuar entre los usuarios y los contenidos. En particular, los alumnos de la Licenciatura en Diseño de la Comunicación Gráfica piensan que el uso de la aplicación Sketchbook en los dispositivos móviles es muy útil durante la realización de las actividades escolares bajo la modalidad del aula invertida. De hecho, la 
Salas-Rueda, R. A., Eslava-Cervantes, A. L. y Prieto-Larios, E.

Análisis sobre el impacto del aula invertida y la tecnología en el proceso educativo sobre el diseño de la comunicación gráfica

mayoría de los estudiantes $(n=13,65.00 \%)$ piensan que las actividades durante la clase facilitaron mucho su rol activo.

La mayoría de los estudiantes ( $\mathrm{n}=13,65.00 \%$ ) consideran que la aplicación Sketchbook mejoró mucho el proceso de aprendizaje. Asimismo, los resultados del aprendizaje automático sobre la Hipótesis 1 son superiores a 0.110 , por lo tanto, el uso de la aplicación Sketchbook en el proceso de aprendizaje influye positivamente el rol activo de los estudiantes bajo la modalidad del aula invertida.

Por otro lado, la mayoría de los estudiantes $(n=11,55.00 \%)$ considera que el uso de dispositivos móviles mejoró mucho el proceso de aprendizaje. Asimismo, los resultados del aprendizaje automático sobre la Hipótesis 2 son superiores a 0.062, por lo tanto, el uso de los dispositivos móviles en el proceso de aprendizaje influye positivamente el rol activo de los estudiantes bajo la modalidad del aula invertida.

El aula invertida transformó el papel del docente y los estudiantes en la asignatura Expresión Formal I por medio de las Tecnologías de la Información y Comunicación. En particular, la aplicación Sketchbook facilita la asimilación del conocimiento por medio del dibujo y uso de los objetos. Asimismo, la tecnología permite crear espacios educativos que son atractivos e interesantes para los estudiantes.

Además, el aula invertida facilita la organización de creativas actividades que favorecen el desarrollo de las competencias en los estudiantes. Por ejemplo, los alumnos de la Licenciatura en Diseño de la Comunicación Gráfica piensan que la aplicación Sketchbook facilita la realización de los trazos y el manejo de imágenes. Incluso, el uso de la tecnología en la asignatura Expresión Formal I incrementa la motivación de los estudiantes durante el proceso de aprendizaje.

\section{CONCLUSIÓN}

El aula invertida facilita la construcción de nuevos espacios donde el alumno aprende los temas de los cursos y desarrolla sus habilidades. En particular, esta investigación transforma el proceso de enseñanza-aprendizaje en la asignatura Expresión Formal I por medio de las herramientas tecnológicas y el aula invertida. Los resultados del aprendizaje automático (regresión lineal) indican que el uso de la aplicación Sketchbook y los dispositivos móviles en el proceso de aprendizaje influyen positivamente el rol activo de los estudiantes.

El papel de los participantes del proceso educativo se transforma bajo la modalidad del aula invertida. Por ejemplo, el docente busca y selecciona los videos sobre la aplicación Sketchbook y prepara las actividades para la sesión presencial. Por otro lado, el alumno participa activamente durante el proceso de enseñanzaaprendizaje por medio de la consulta de los videos antes de la clase y el uso de la tecnología en el salón de clases. 
Salas-Rueda, R. A., Eslava-Cervantes, A. L. y Prieto-Larios, E.

Análisis sobre el impacto del aula invertida y la tecnología en el proceso educativo sobre el diseño de la comunicación gráfica

Los alumnos de la Licenciatura en Diseño de la Comunicación Gráfica piensan que el uso de la aplicación Sketchbook en los dispositivos móviles facilita la asimilación del conocimiento sobre el trazo del rostro humano y desarrolla las habilidades relacionadas con el dibujo. Las limitaciones de esta investigación son el tamaño de la muestra y el uso de la aplicación Sketchbook en los dispositivos móviles. Por lo tanto, las futuras investigaciones pueden analizar el impacto del aula invertida en grupos que presenten un mayor número de estudiantes y evaluar la incorporación de otras herramientas tecnológicas como las redes sociales y la realidad aumentada antes, durante y después de la sesión presencial.

Esta investigación mixta recomienda el uso del aula invertida en el campo educativo debido a que este modelo pedagógico, junto con la tecnología, facilitan el rol activo de los estudiantes durante el proceso de aprendizaje. Por último, los docentes pueden innovar las actividades escolares, mejorar las condiciones de enseñanza-aprendizaje, fomentar la participación activa de los estudiantes, facilitar la asimilación del conocimiento y desarrollar las habilidades de los participantes del proceso educativo por medio del aula invertida.

\section{REFERENCIAS}

Bakla, A. (2018). Learner-generated materials in a flipped pronunciation class: A sequential explanatory mixed-methods study. Computers $\mathcal{E}$ Education, 125, 14-38. https://doi.org/10.1016/j.compedu.2018.05.017

Barbour, C., \& Schuessler, J. B. (2019). A preliminary framework to guide implementation of The Flipped Classroom Method in nursing education. Nurse Education in Practice, 34, 36-42. https://doi.org/10.1016/j.nepr.2018.11.001

Busebaia, T. J. A. \& John, B. (2020). Can flipped classroom enhance class engagement and academic performance among undergraduate pediatric nursing students? A mixed-methods study. Research and Practice in Technology Enhanced Learning, 15, 116.

Gamboa-Rodríguez, F. (2015). Diseño de espacios colaborativos interactivos para el aprendizaje. En J. Zubieta-García y C. Rama-Vitale (Eds.), La educación a distancia en México: Una nueva realidad universitaria (pp. 201-212), México, UNAM.

He, W., Holton, A. J., \& Farkas, G. (2018). Impact of partially flipped instruction on immediate and subsequent course performance in a large undergraduate chemistry course. Computers $\mathcal{E}$ Education, 125, 120-131. https://doi.org/10.1016/j.compedu.2018.05.020

Hermsen, J. L., Mokadam, N. A., \& Verrier, E. D. (2019). Flipping the Classroom: How to Optimize Learning in the Didactic Setting. Thoracic Surgery Clinics, 29(3), 279-284. 
Salas-Rueda, R. A., Eslava-Cervantes, A. L. y Prieto-Larios, E.

Análisis sobre el impacto del aula invertida y la tecnología en el proceso educativo sobre el diseño de la comunicación gráfica

Hew, K. F. \& Lo, C. K. (2018). Flipped classroom improves student learning in health professions education: a meta-analysis. BMC Medical Education, 18, 1-12.

Rotellar, C. \& Cain, J. (2016). Research, Perspectives, and Recommendations on Implementing the Flipped Classroom. American Journal of Pharmaceutical Education, 80(2), 1-9.

Rui, Z., Lian-Rui, X., Rong-Zheng, Y., Jing, Z., Xue-Hong, W., \& Chuan, Z. (2017). Friend or Foe? Flipped Classroom for Undergraduate Electrocardiogram Learning: a Randomized Controlled Study. BMC Medical Education, 17, 1-19.

Salas-Rueda, R. A. (2015). Interfaz web usable: herramienta tecnológica para el proceso de enseñanza-aprendizaje. Revista de Comunicación de la SEECI, 36, 148-177. http://dx.doi.org/10.15198/seeci.2015.36.148-177

Salas-Rueda, R. A. (2018). Analysis on the Use of Continuous Improvement, Technology and Flipped Classroom in the Teaching-Learning Process by means of Data Science. Online Journal of Communication and Media Technologies, 8(4), 325-343. https://doi.org/10.12973/ojcmt/3955

Salas-Rueda, R. A. (2020). Use of the flipped classroom to design creative and active activities in the field of computer science. Creativity studies, 13(1), 136-151. https://doi.org/10.3846/cs.2020.10336

Tague, J. \& Czocher, J. (2016). A Theoretical Approach to Ensuring Instructional and Curricular Coherence in the Flipped Classroom Model of a Differential Equations Course. International Journal of Research in Undergraduate Mathematics Education, 2, 223-245.

Uskoković, V. (2018). Flipping the flipped: the co-creational classroom. Research and Practice in Technology Enhanced Learning, 13, 1-24.

Zainuddin, Z. (2018). Students' learning performance and perceived motivation in gamified flipped-class instruction. Computers $\mathcal{E}$ Education, 126, 75-88. https://doi.org/10.1016/j.compedu.2018.07.003

\section{AUTORES:}

\section{Ricardo-Adán Salas-Rueda}

Doctor en Diseño de Nuevas Tecnologías. Investigador de tiempo completo en el Instituto de Ciencias Aplicadas y Tecnología, Universidad Nacional Autónoma de México. Investigador Nacional SNI nivel 1 (2019-2021).

ricardo.salas@icat.unam.mx

Orcid ID: http:// orcid.org/0000-0002-4188-4610 
Salas-Rueda, R. A., Eslava-Cervantes, A. L. y Prieto-Larios, E.

Análisis sobre el impacto del aula invertida y la tecnología en el proceso educativo sobre el diseño de la comunicación gráfica

Google Scholar: https://scholar.google.com/citations?hl=es\&user=avsvdDUAAAAJ

\section{Ana-Libia Eslava-Cervantes}

Instituto de Ciencias Aplicadas y Tecnología, Universidad Nacional Autónoma de México. México.

libia.eslava@icat.unam.mx

Orcid ID: https:/ / orcid.org/0000-0002-7420-3412

\section{Estefanía Prieto-Larios}

Universidad Nacional Autónoma de México. México.

estefaníaprietolarios@gmail.com

Orcid ID: $\underline{\text { https://orcid.org/0000-0002-8140-2778 }}$ 